\title{
Sable et préhistoire saharienne
}

Entretien avec Robert Vernet

Sand, Prehistoric Man and the Archaeologist in the Sahara

Robert Vernet, Sébastien Boulay et Marie-Luce Gélard

\section{(2) OpenEdition}

Journals

Édition électronique

URL : https://journals.openedition.org/tc/7336

DOI : $10.4000 /$ tc. 7336

ISSN : 1952-420X

Éditeur

Éditions de l'EHESS

Édition imprimée

Date de publication : 15 décembre 2013

Pagination : 286-303

ISBN : 978-2-7351-1654-6

ISSN : 0248-6016

Référence électronique

Robert Vernet, Sébastien Boulay et Marie-Luce Gélard, «Sable et préhistoire saharienne », Techniques \& Culture [En ligne], 61 | 2013, mis en ligne le 15 décembre 2016, consulté le 29 septembre 2022. URL http://journals.openedition.org/tc/7336 ; DOI : https://doi.org/10.4000/tc.7336 


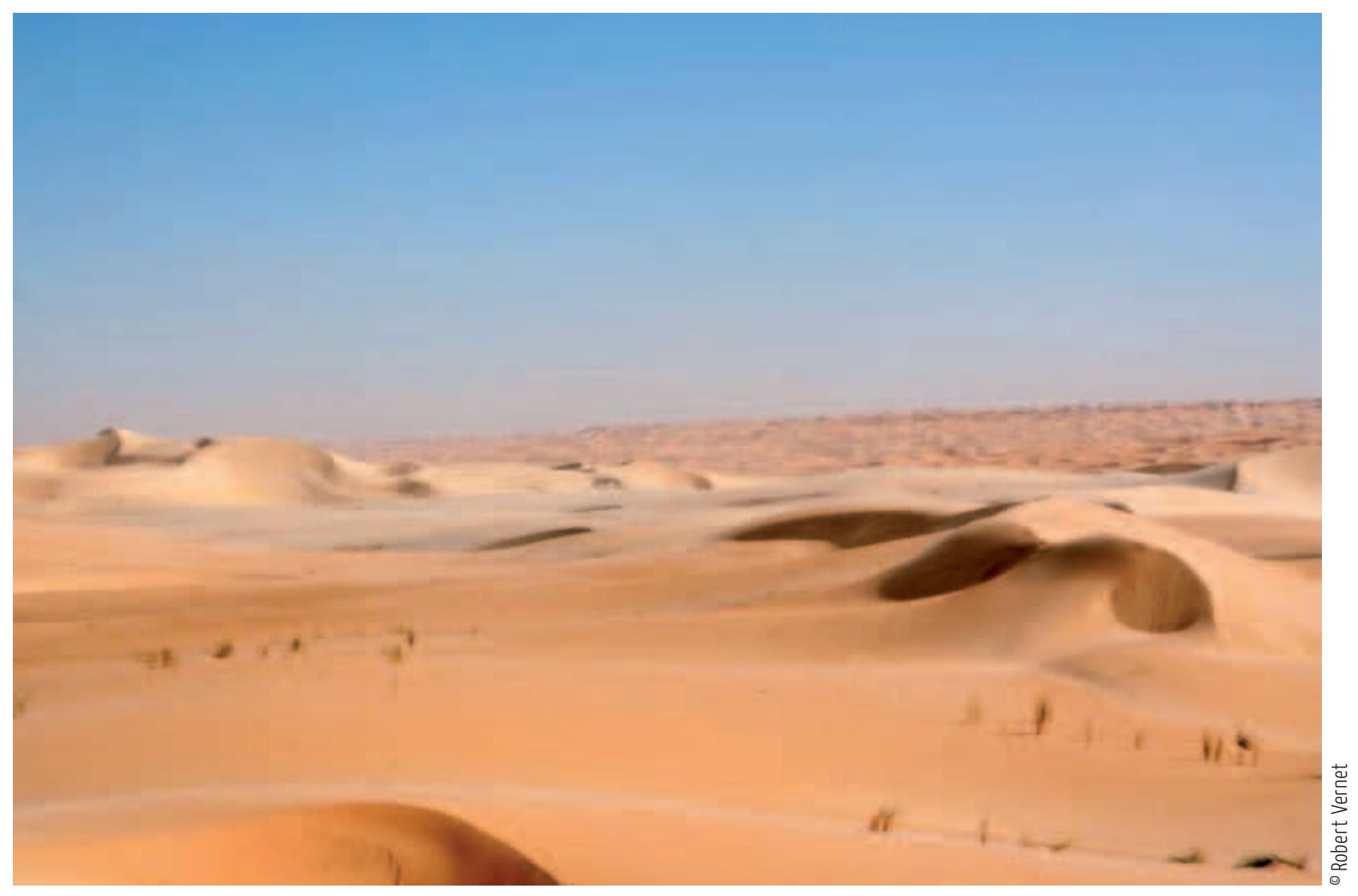




\section{SABLE ET PRÉHISTOIRE SAHARIENNE}

\section{Entretien avec Robert Vernet}

Sébastien Boulay et Marie-Luce Gélard: Nous souhaitions aborder deux axes de réflexion avec vous: premièrement, comment l'homme préhistorique saharien gérait cette matière, le sable, omniprésente et envahissante au quotidien, matériellement et symboliquement; deuxièmement, quelles conditions de recherche et de méthode le sable impose-t-il à l'archéologue. Mais peut-être est-il important de commencer par quelques mots très généraux sur la présence de l'homme au Sahara durant la Préhistoire?

Robert Vernet: Cette question est en effet essentielle: aujourd'hui on voit le Sahara comme un grand tas de sable. Mais lorsque l'homme est présent au Sahara, le sable n'est pas forcément là: le Sahara est un désert où l'homme n'est véritablement présent que lorsqu'il y a une période pluvieuse, autrement dit lorsque le sable est couvert de végétation. On est actuellement, depuis quelques millénaires (trois à six selon les régions), dans une période aride - pas totalement d'ailleurs, puisqu'il y a eu des épisodes où le Sahara fut absolument vide - ce qui n'est pas le cas aujourd'hui. Mais ce que vous me demandez, c'est de parler de l'homme préhistorique qui, lui, vit dans un milieu où il peut alternativement chasser l'éléphant, la girafe, voire l'hippopotame, ou seulement la gazelle et l'autruche; où il peut pêcher de grands poissons ou élever des vaches; et, sur la fin, dans les oasis et le sud du Sahara, pratiquer l'agriculture. Donc dans un milieu où le sable n'est pas l'élément principal.

En revanche, c'est dans les périodes de début et de déclin, donc de retour à des conditions très sèches et dans les régions où le climat est toujours resté très aride que le sable est omniprésent. L'aridité est une notion excessivement relative: aujourd'hui, dans les zones où il tombe $5 \mathrm{~mm}$ par an, c'est le paradis s'il tombe dix ans de suite $50 \mathrm{~mm}$. À l'inverse, dans le Sahara méridional, les années où il ne tombe pas $250 \mathrm{~mm}$, c'est-à-dire où l'on ne peut pas cultiver dans les dépressions, c'est une catastrophe. Donc cette relativité de la notion d'aridité fait qu'il est difficile de généraliser. Cela dit, même dans les périodes de prospérité, le sable est présent sous la végétation. Or, le sable étant perméable, l'eau percole et ne perdure que lorsqu'une couche imperméable est présente sous le sable. De ce fait, dans une bonne partie du Sahara, même dans les périodes pluvieuses, il n'y a pas d'eau. Elle est située trop en profondeur pour qu'on puisse aller la chercher. Un exemple en est l'erg du Trârza, en Mauritanie, où les puits 


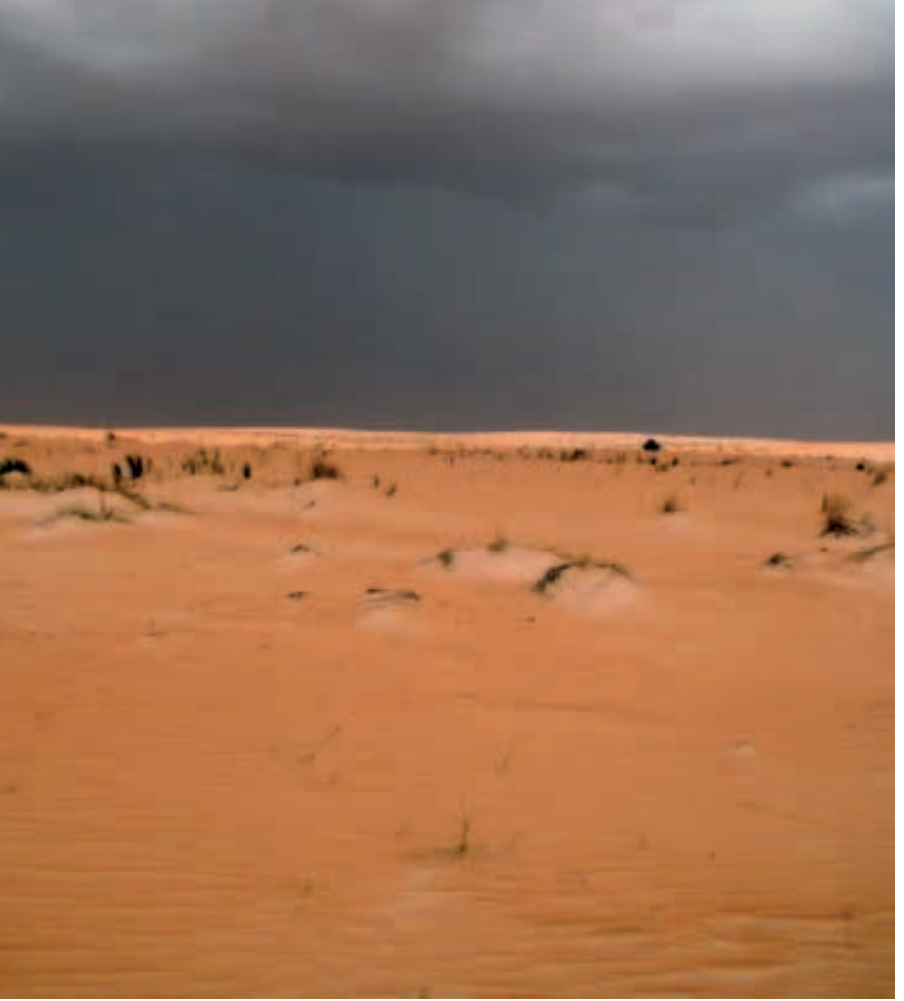

Le sable à perte de vue et l'orage qui menace

Dans quelques jours, le sable sera caché par des millions de plantules, qui fleuriront très vite (erg Waran).

actuels se trouvent à plus de 100 mètres, donc à une profondeur totalement inaccessible à l'homme préhistorique.

S.B. : Durant la Préhistoire, les hommes s'alimentent donc en eau à partir de mares?

R.V.: ... Des réseaux hydrographiques qui existent à cette période, des mares permanentes, des marigots temporaires, des sources, des gueltas: à l'époque les ressources hydriques sahariennes ressemblent à celles des régions « subsahariennes » d'aujourd'hui.

M.-L.G. : Pouvez-vous nous préciser quand commence et s'achève la Préhistoire au Sahara?

R.V.: Probablement depuis plus d'un million d'années jusqu'à 2000 avant aujourd'hui (BP = before present), mais pas partout au Sahara en même temps. Surtout, les informations sont insuffisantes pour la période située avant le Néolithique (qui débute dans certaines régions du Sahara il y a 10000 ans, mais seulement vers 7500 en Mauritanie). Je vais pour ma part évoquer essentiellement le Néolithique moyen, voire récent.

M.-L.G.: Comment s'est manifestée la fin du Néolithique: y a-t-il eu rupture nette dans les modes de vie ou continuité avec la période qui a suivi?

R.V.: Il est vrai qu'il y a du métal à partir de $3500 \mathrm{BP}$ à peu près, que l'agriculture arrive dans le Sahara méridional à peu près à la même époque (sans doute plus anciennement dans certaines zones, la question est ouverte), mais le mode de vie et probablement les mentalités des habitants continuent à être néolithiques: un mélange variable et éventuellement réversible de chasse, cueillette, élevage et parfois agriculture, avec apparition progressive d'objets en métal dans un milieu toujours néolithique... Je ne vois pas, dans les régions où je travaille, de rupture avant qu'apparaissent des villes. On a, par exemple, la culture du dhar Tichit (4000-2000 BP), où existent plus de 500 villages, qui reste pour moi complètement néolithique: il s'agit d'un mode d'installation de type rural, et non de type urbain, même s'il y a de très gros villages. En fait, le mode de vie néolithique persiste dans la plupart des régions sahariennes jusqu'à ce qu'il ne soit plus vraiment possible d'y vivre autrement que comme les petits éleveurs d'aujourd'hui, en interactions avec les populations oasiennes.

S.B. : Peut-on considérer que l'arrivée du dromadaire a reconfiguré l'implantation humaine dans la zone et a permis à des groupes d'aller investir des espaces «sableux »?

R.V.: Le dromadaire est à la fois conséquence et cause... Lorsqu'il n'est plus possible d'élever vaches ou moutons, on passe à la chèvre... Et, à un moment, il n'est plus possible d'utiliser un autre moyen de transport que le dromadaire, qui remplace le bouf porteur et le cheval. La sécheresse est là, à laquelle seules certaines populations peuvent, ou veulent, adapter leur mode de vie.

S.B.: Peut-on dater assez précisément l'aridification?

R.V.: Je viens d'écrire un article sur cette question avec Jean Maley (2013). Cela dépend des zones. Dans la Majabât el-Kubrâ' (zone la plus aride et inhospitalière du Sahara 
mauritanien, au centre-est du pays), les traces humaines sont relativement excentrées par rapport au cœur de cette région très aride, mais vers $2800 \mathrm{BP}$, des éleveurs de bovins viennent y passer l'hivernage. Ensuite, à partir de 2500 BP, les conditions de vie dans ces zones deviennent très difficiles. La saison sèche est de plus en plus longue, alors qu'on ne dispose pas encore du dromadaire, qui arrive sans doute il y a moins de 2000 ans. Au début de notre ère, le paysage est désertique, avec néanmoins des épisodes pluvieux plus longs qu'aujourd'hui. Cette période voit effectivement une réorganisation de l'espace, de l'économie et donc, fatalement, du mode de vie.

M.-L.G. : Pour en venir plus précisément au thème de ce numéro, quelles hypothèses peut-on faire sur le rapport qu'entretenaient ces Sahariens de la Préhistoire avec le sable?

R.V.: Dans les périodes ou les lieux les plus favorables à la vie humaine, les archéologues ne peuvent pas dire grand-chose sur le rapport des hommes de la Préhistoire au sable. Il faut en effet imaginer des dunes de sable totalement vêtues de végétation jusqu'à des latitudes de 22 à $25^{\circ}$ Nord. Les hommes préhistoriques préféraient s'installer dans des endroits sableux, confortables, propres, plutôt que sur des rochers ou des tas de coquilles. En outre, les dunes ont l'avantage de permettre de choisir le versant sur lequel on va s'installer, et donc, de ce fait, d'être à l'abri du vent. Il y a un exemple frappant en Mauritanie où, avant le Néolithique, il y a peut-être 9000 ans, une culture appelée épipaléolithique a installé systématiquement ses campements sur le versant nord-est des dunes, ce qui signifie qu'à cette époque les vents dominants venaient de la direction opposée, donc du sudouest. Or, ce qui est étonnant c'est que, deux ou trois millénaires plus tard, des néolithiques vont s'installer sur l'autre versant des dunes, donc au sud-ouest.

Ensuite, les villages ou les campements néolithiques étaient installés sur le flanc et le haut des dunes, contrairement à ce que font les nomades d'aujourd'hui qui ne se mettent pas en hauteur. Cela permettait de surveiller la plaine - donc les gens qui arrivaient - et le bétail. Cela permettait aussi de répartir spatialement les habitations du village, les lieux de cuisson (cuisine ou céramique) ou de travail.

S.B. : Pour rester sur la question du confort et de l'habitat, a-t-on des éléments sur cet habitat du Néolithique et de la fin du Néolithique, et en particulier sur cet abri minimal qu'est le paravent, que l'on utilise beaucoup au Sahara?

R.V.: Il faut évidemment savoir qu'il y a des régions du Sahara montagneuses et rocheuses, où les hommes ont privilégié des habitats entre les rochers, dans des abris sous roche ou des grottes. En revanche, le Sahara méridional, qui est très peuplé au Néolithique moyen et récent, a beaucoup plus utilisé le paravent. En ce qui concerne les techniques de protection contre le sable, le mieux lorsqu'il n'y a rien de construit en dur c'est la tente. Mais des tentes proprement dites, on n'en a pas vraiment de traces dans l'art rupestre. En revanche, on y trouve des traces de cases démontables comme celles des Peuls, encore visibles aujourd'hui au Sahel.

\section{Lambeau d'un paléolac dans un interdunaire de l'erg Waran (N.E. de l'Adrar)}

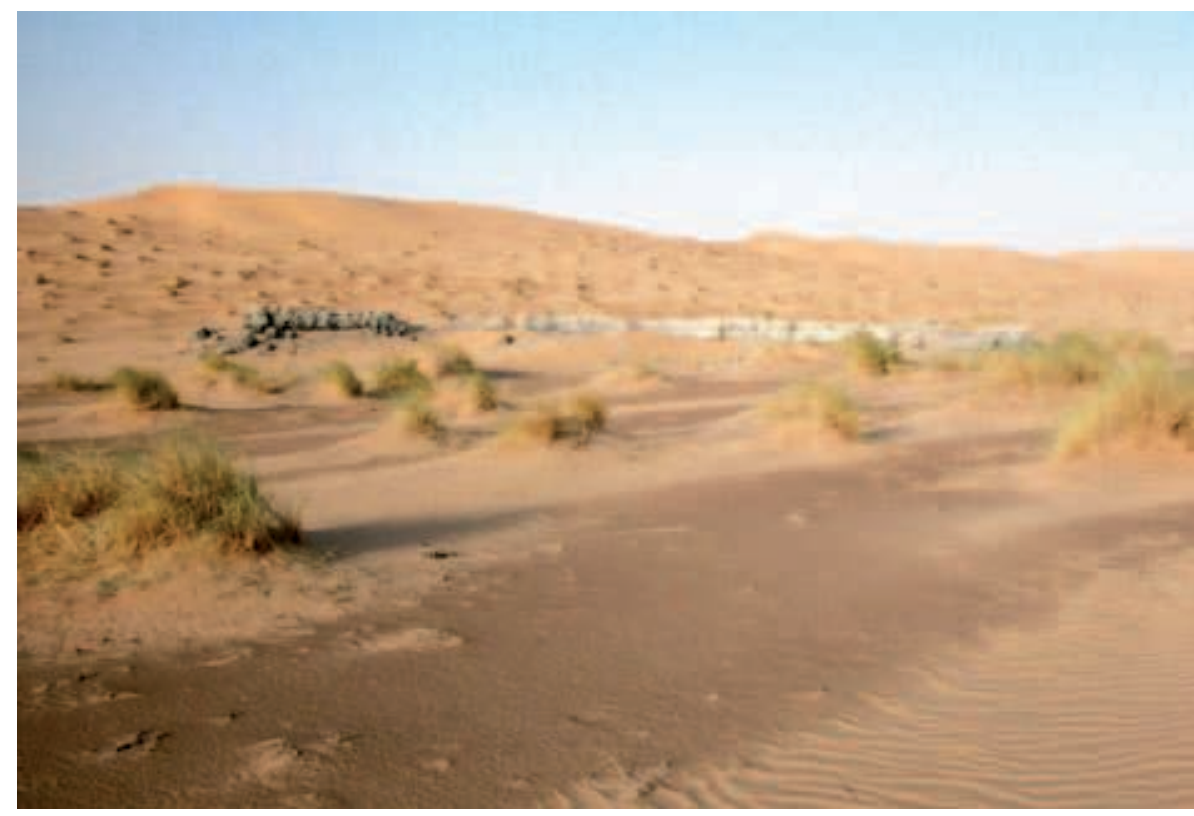


Trois exemples de réoccupation de dunes sur le golfe d'Arguin

Les vents ont changé de direction entre l'Épipaléolithique et le Néolithique.

Sur FA 38, un sondage permet de retrouver les deux périodes, dans une modeste stratigraphie.
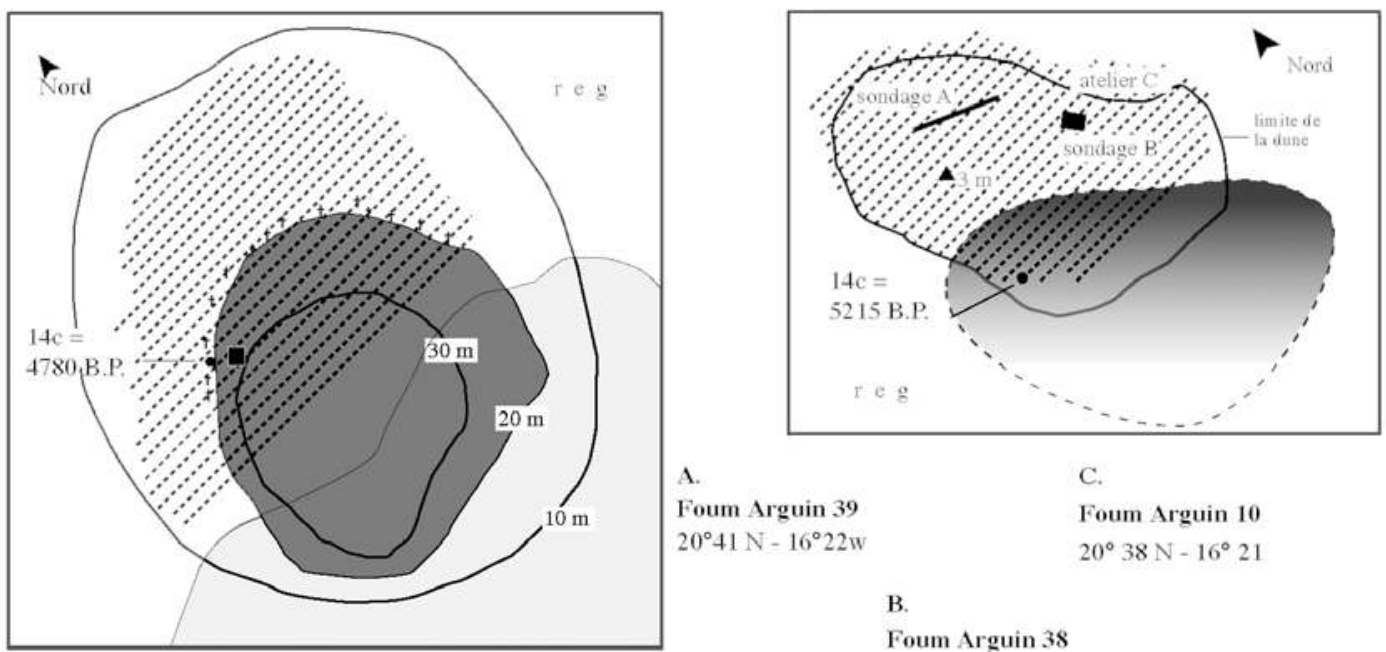

A.

Foum Arguin 39

$20^{\circ}+1 \mathrm{~N}-16^{\circ} 22 \mathrm{~W}$

\section{C.}

Foum Arguin 10

$20^{\circ} 38 \mathrm{~N}-16^{\circ} 21$
B.

Foum Arguin 38

$20^{\circ} 40 \mathrm{~N}-16^{\circ} 23 \mathrm{w}$

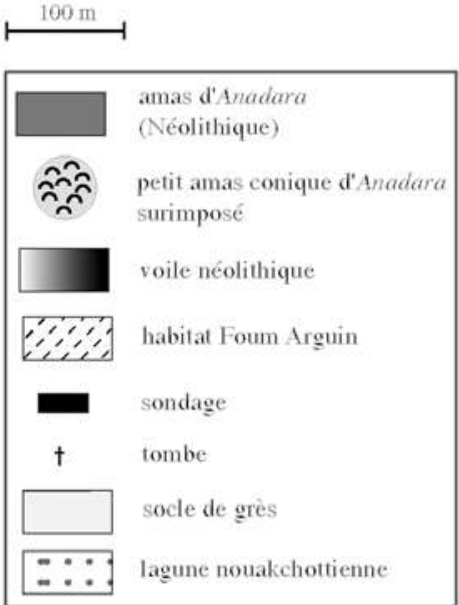
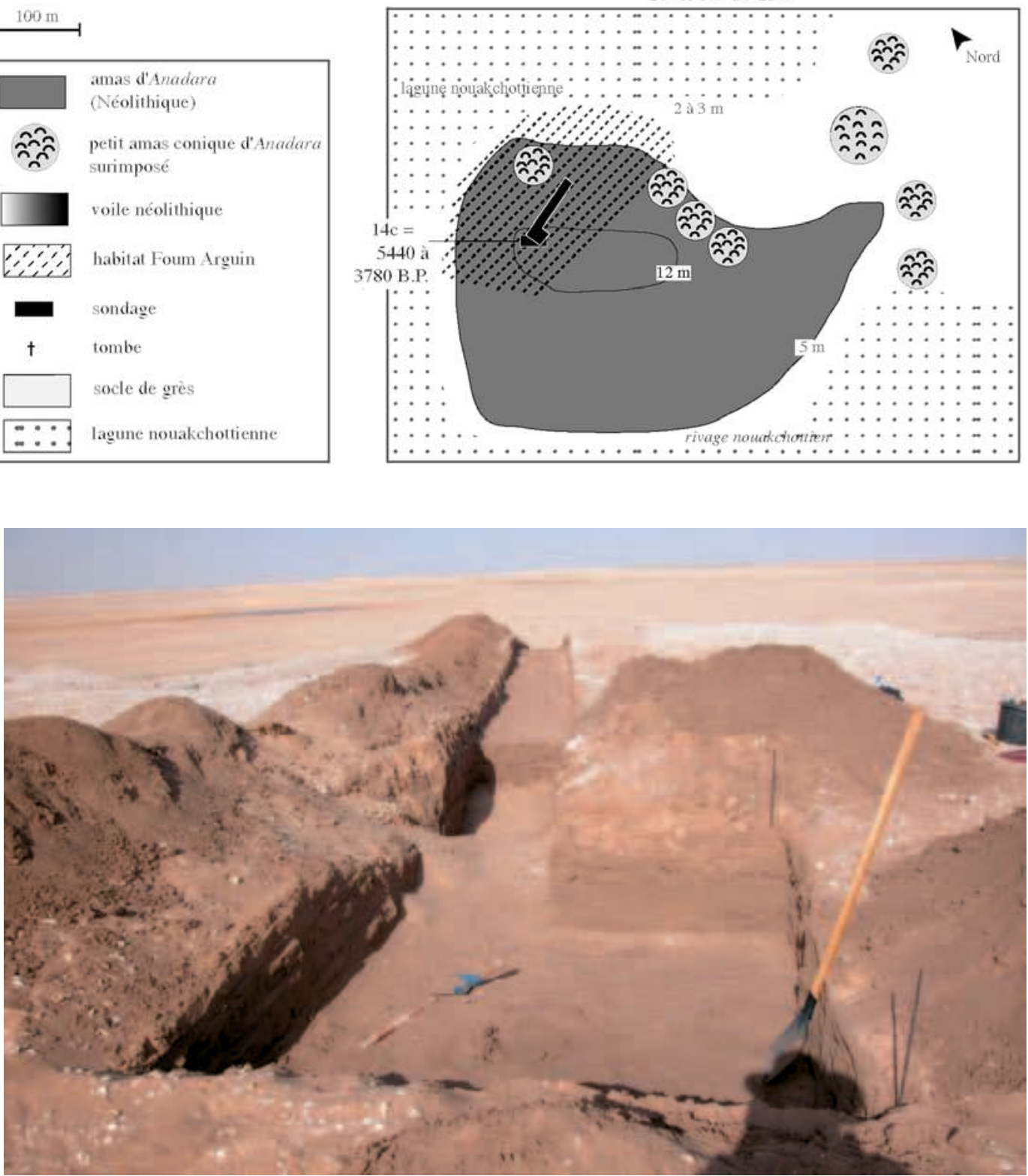
Dans les fouilles en zone sableuse, les stratigraphies sont rarissimes car il n'y a aucune chance de trouver, comme en Europe, des niveaux d'installation successifs - et avec des trous de poteaux (Figure 3b). En 2012, j'ai découvert près de Boujertala (Nord-Est mauritanien) un atelier de fabrication de grattoirs de $10 \mathrm{~m}$ de diamètre. On peut imaginer que l'artisan travaillait sous une case ou à l'abri d'un paravent. Mais on avait pas, pendant la Préhistoire, trente-six manières de se protéger du soleil, du vent et du sable et que cela n’a pas changé jusqu'à nos jours, où les nomades ont encore recours à des bâches, souvent en plastique, pour se protéger des éléments.

\section{Permanence du mode d'habitat de plein air dans le Sahara central}

Ci-contre,

Croquis des peintures du même site et reconstitution de la tente bovidienne, (in Brigitte Khan Majliss, 1978 : 366).

Ci-dessous,

A l'aide d'arceaux en bois,

les femmes touarègues de l'Aïr (Niger) procèdent aujourd'hui, de la même manière qu'il y a 7000 ans, au montage de la case à velum en nattes, avec beaucoup de soin, (in Khan Majliss B., 1978 : 367). peut juste imaginer, pas plus. Ce qui est évident c'est qu'il n'y

M.-L.G.: Et qu'en était-il des techniques hydrauliques et d'exhaure à l'époque?

R.V.: Avec les techniques que l'on connaît à l'époque, on ne pouvait pas creuser très profond. Des puits néolithiques ont été trouvés entre 3 et $5 \mathrm{~m}$ de profondeur au Soudan et il existe une peinture rupestre du Tassili, assez récente mais néolithique quand même, qui présente une vache et un abreuvoir (Figure 5). De toutes manières, un puits qui a 3000 ou 4000 ans, s'il n'a pas été utilisé en permanence, disparaîtra dès la première période de sécheresse.
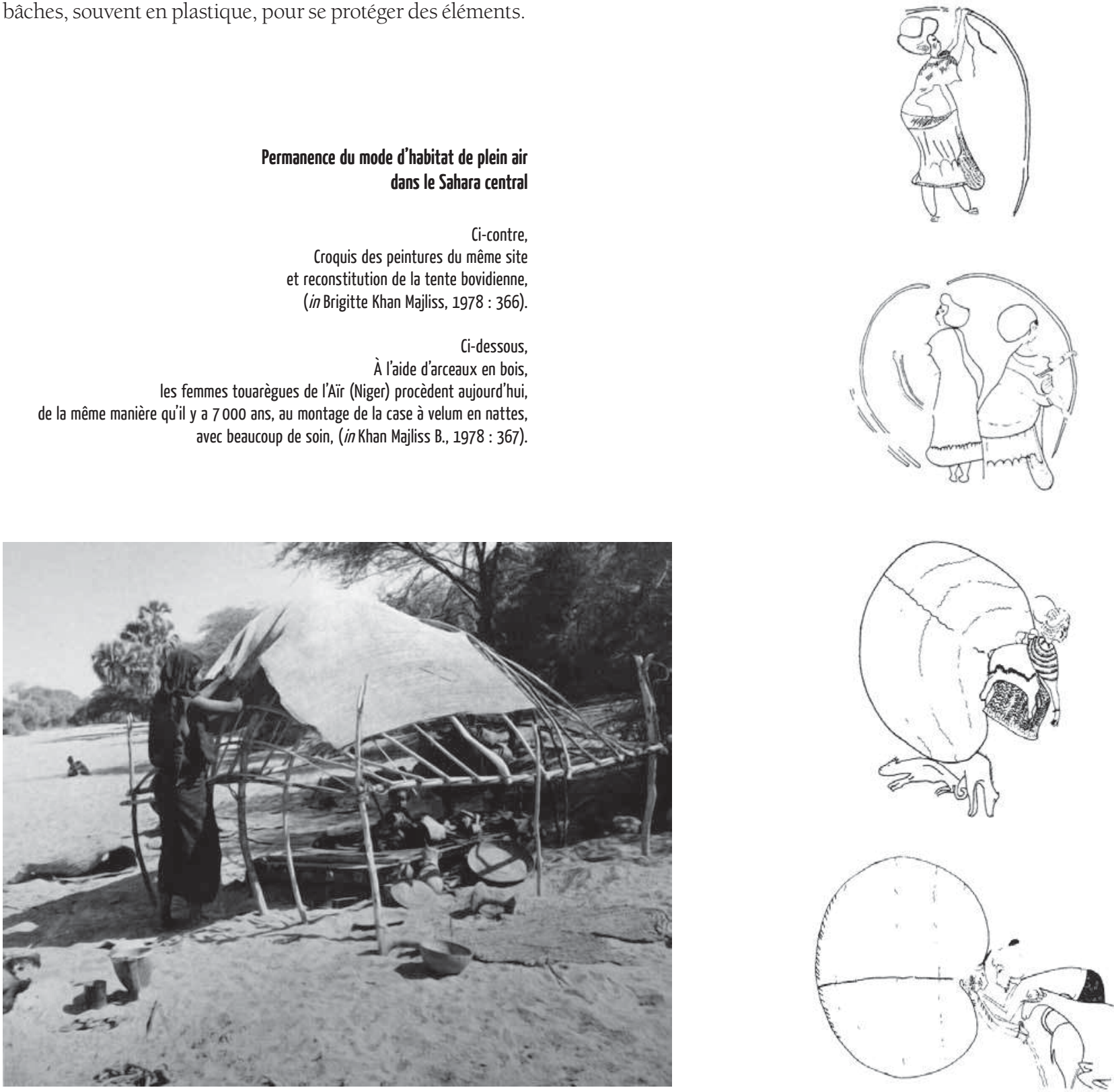
S.B.: Venons-en à présent aux pratiques d'inhumation, que peut-on dire de la façon dont le corps du défunt était enfoui dans le sable?

R.V.: J'ai un exemple remarquable à ce sujet: c'est celui de Khatt Lemaîteg dans l'Amatlîch (erg de Mauritanie occidentale), qui est daté entre 3500 et 2900 ans avant aujourd'hui (Bathily 1998). On a retrouvé plus de cent quatre-vingts tombes. Quand on les a découvertes, à la fin des années 1970, de forts vents de sable venaient juste de les dégager. Les habitants du site étaient inhumés dans le sable. La totalité de ces tombes se trouvait au milieu d'un village néolithique immense - plus de cinq hectares - et d'une richesse archéologique exceptionnelle, malgré le pillage. Et on a retrouvé la totalité de ces tombes au milieu des déchets du site. J'imagine mal que ces tombes ne soient pas d'un type assez classique au Sahel, c'est-à-dire sous des cases. Mais les tombes étaient dans le sable. Le désert revenu, le village a disparu. Aucun sol ne protégeant plus les tombes des vents, des pluies rares mais violentes et des animaux, les squelettes ont fini par se retrouver en surface. Il ne faut alors que quelques semaines pour que le sable et ses effets abrasifs détruisent les inhumations.

Dans ce village de Khatt Lemaiteg, les défunts avaient été souvent enterrés assis, probablement dans un sac de cuir ou ficelés.

\section{Scène de puits}

Tassili n’Ajjer, environ 3500 BP.
M.-L.G. : N'y a-t-il aucune trace de pratiques de dessiccation des corps et de conservation, comme en Égypte ancienne par exemple, avec des corps parfaitement momifiés?

R.V.: Non, parce que cette région ouest-saharienne n'était pas et n'est pas suffisamment aride pour que l'on puisse recourir à ces techniques. L'Égypte des défunts momifiés, ce sont 5 à $10 \mathrm{~mm}$ de précipitations en moyenne par an depuis 5000 ans.

M.-L.G.: On n'a donc rien d'équivalent ailleurs au Sahara? R.V.: On a un exemple à Tin Hanakaten (Tassili algérien) d'une momification partielle. Le mort est un enfant négroïde, qui a 9000 ans, et qui portait encore des traces de peau et de cheveux. Il vient de faire l'objet d'une publication qui apporte de nouvelles précisions (Heim et al. 2011). Une autre momification partielle provient de la Tadrart libyenne et a 6200 ans. Je le répète, pour que la conservation se fasse, la condition sine qua non est une aridité complète, ce qui n’a pas été le cas partout dans le Sahara. Dans la région de Khatt Lemaiteg, il y a encore aujourd'hui des terrains de culture les bonnes années. C'est d'ailleurs un problème pour les archéologues: on a très peu de restes humains dès lors qu'il pleut, même très épisodiquement, au Sahara.

Plus près de Nouakchott, l'extraordinaire cimetière d'Ain en Nouss, daté de 4500 ans, comporte plus de cent cinquante tombes. Les morts y ont été enterrés dans le sable, sans la moindre protection visible. Il ne reste que quelques dents et des perles en coquilles marines sur certaines tombes. Les perles de ces colliers se sont soudées ensemble au cours de la destruction organique des corps.

S.B.: Que voulez-vous dire par « aucune protection visible »? R.V.: Et bien parce qu'il existe, y compris à Khatt Lemaiteg, quelques rares exemples de tombes dans le sable, qui sont en fait des monticules de sable, sur lesquels ont été placés des plaques de pierre et des graviers pour les protéger. Mais le résultat est fragile et n'a d'intérêt que dans les zones où la pierre est très rare. Mais on peut penser que beaucoup ont disparu.

S.B.: Quand les archéologues du Sahara parlent de tombes, de quoi s’agit-il généralement?

R.V.: Il s'agit essentiellement des traces du mort, donc os, dents et mobilier funéraire. Il y a un exemple récent dans le Nord Niger, à Gobero où, à défaut de momies, des squelettes se sont préservés parfaitement, dans un sable pourtant argileux et non quartzeux, donc a priori moins favorable à la conservation (Sereno, 2008). C'est beaucoup plus rare en Mauritanie. 
M.-L.G.: A-t-on autre chose que des peintures rupestres en guise de témoignages sur l'habitat en milieu ouvert? R.V.: Il m'est arrivé de trouver sur le cordon dunaire de la Dhraïna une dizaine de poteries alignées le fond en l'air sur moins de dix mètres de long - autrement dit un rangement pour l'année ou la saison. Il est très vraisemblable que ces poteries, âgées de 4500 ans, se trouvaient à l'abri d'un paravent en matière végétale ou en peau. Il est aussi assez émouvant de penser que ces éleveurs nomades ont à l'époque quitté leur campement en se disant qu'ils reviendraient à la saison des pluies suivante et qu'ils ne sont en fait jamais revenus!

Mais revenons à l'Ain en Nouss où les défunts ont été enterrés dans le sable dur d'une dune ancienne, surmontée par des dunes vives, ce qui a protégé les tombes jusqu'à 2003. Il a fallu une violente période de vents de sable cette année-là pour qu'un collectionneur du dimanche vienne me chercher en me disant de venir voir. Nous nous sommes retrouvés dans un creux étroit $(10 \times 20 \mathrm{~m})$ où se trouvaient 150 tombes, accompagnées de 500 poteries. Les plus belles poteries du Musée National de Nouakchott en proviennent. Nous sommes venus travailler trois jours d'affilée, dans une tempête ininterrompue, qui menaçait d'engloutir mon véhicule... D'ailleurs, la semaine suivante, seul le GPS m'a permis de retrouver le site: il y avait à nouveau dix mètres de sable dessus! Cela peut vous donner une idée des conditions très délicates et précaires dans lesquelles les archéologues travaillent au Sahara.

Nous avons notamment découvert sur ce site quatre types de céramique totalement différents, sans rien en commun. Les défunts étaient enterrés ensemble sur un espace réduit. Homogénéité du cimetière, hétérogénéité totale des hommes qui étaient enterrés là! Apparaissaient quatre « cultures » ou groupes humains différents, représentés par ces quatre céramiques différentes, alors que ces groupes ne se montrent en général pas ensemble ailleurs dans la région. Et là on les retrouve dans un cimetière homogène, présentant une même couche d'occupation. Ils ont mis des gens différents dans le même cimetière en même temps! Ils sont d'ailleurs enterrés exactement de la même manière (Vernet \& Boulanger 2006).

Dans le sable, ont été retrouvés les restes des morts, mais aussi ce qui a été enterré avec eux, plus souvent des perles que des outils. L'exemple d'une tombe un peu plus récente sur l'île de Tidra (golfe d'Arguin) est exceptionnel: deux personnes y ont été enterrées ensemble avec chacune plusieurs milliers de perles en fragments d'œuf d'autruche ou dans une petite coquille marine qu'on appelle un dentale, et qui a la forme d'un tube légèrement coudé. L'homme est enterré avec un cache-sexe confectionné avec

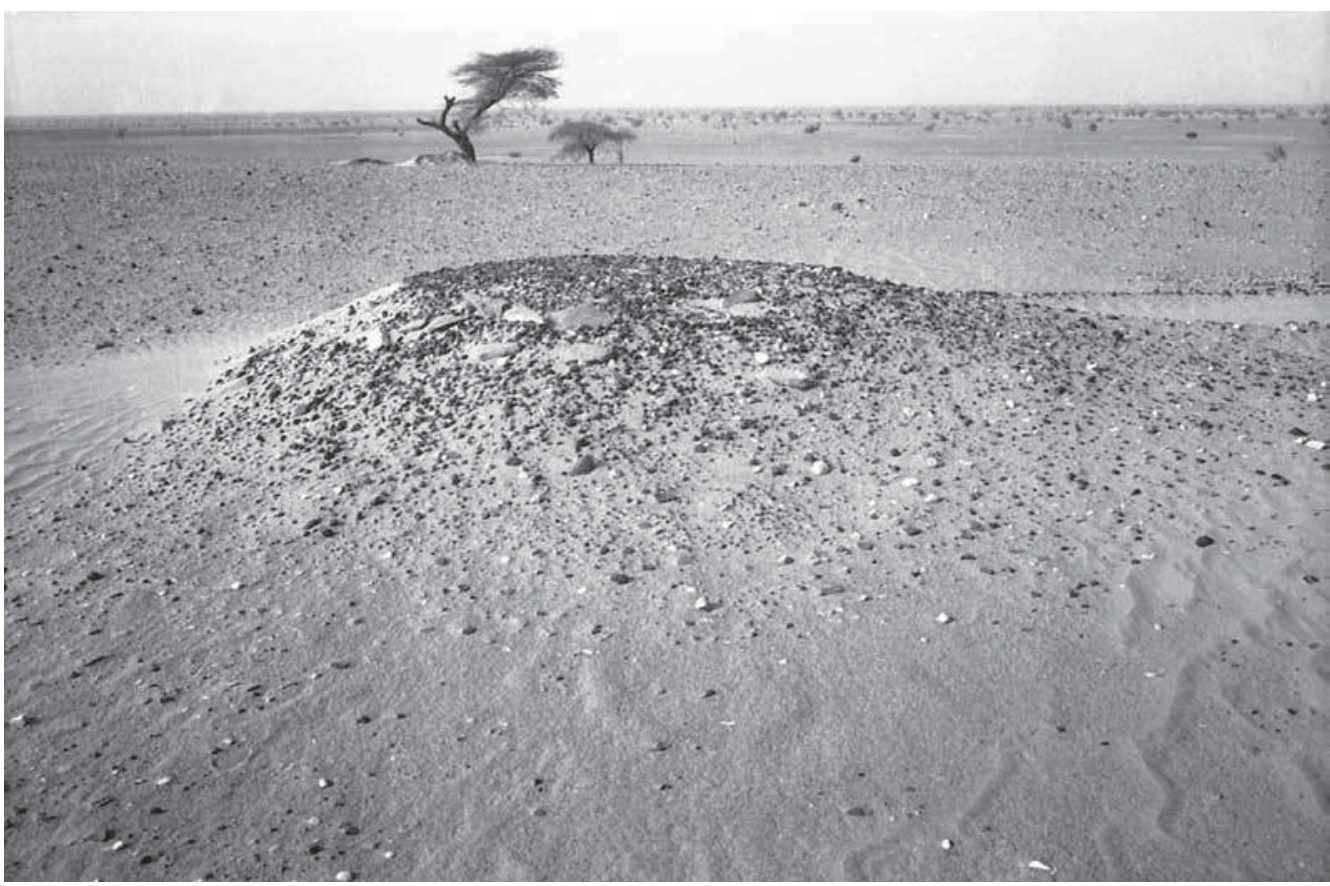

Tumulus de sable de Khatt Lemaïteg, couvert de graviers et de fragments de meules 

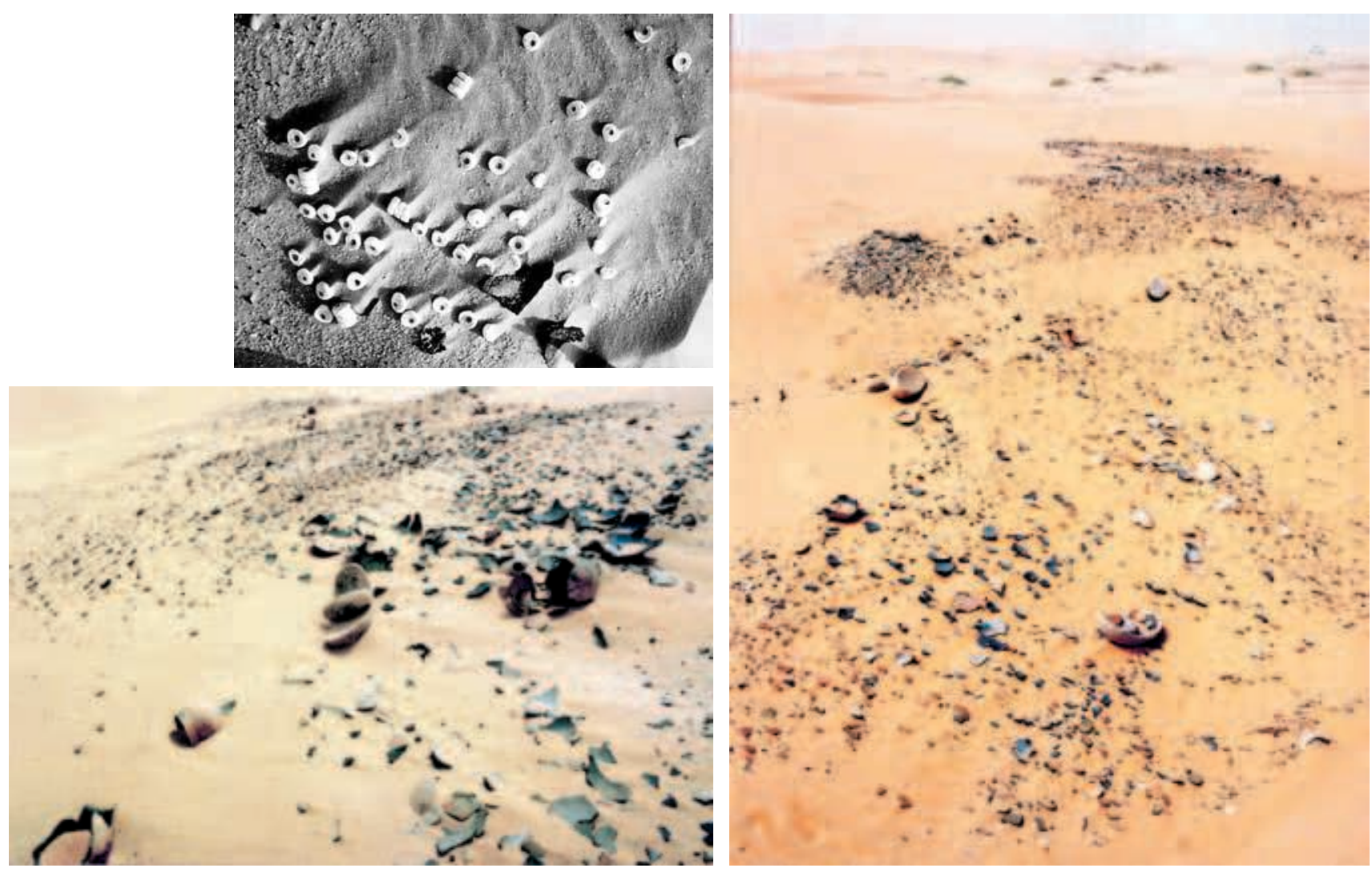

Cimetière de l'Aïn en Nouss (4 500 calBP) : une fenêtre ouverte par le vent entre les dunes pendant quelques jours en mars 2003. Figures a ci-dessus et b ci-contre. Un collier : il était associé à quelques dents, seuls témoins d'une inhumation. Figure c, en haut à gauche.

des dentales, qui ressemble à un slip à mailles ouvertes, remarquablement conservé; la femme avec une courte jupe également en dentales.

Ce que l'on retrouve également assez souvent sur les tombes ce sont des poteries. Par exemple, sur le site d'Ain en Nouss, il y a sur certaines tombes jusqu'à trente ou quarante poteries entières. On a mis dans ces tombes (ou sur ces tombes: on ne le sait que rarement) des poteries entières (mais ont-elles été cassées au moment de l'inhumation?) et non des morceaux de poteries (Figure p. 12; ci-dessus a, b). C'était aussi une façon de protéger le défunt. Dans les amas coquilliers, c'est plus facile puisqu'effectivement les inhumations se font dans le sable qui se trouve sous la couche de coquilles, qui protège bien le défunt.

M.-L.G. : Les poteries avaient-elles une seule fonction de protection des corps? N'avaient-elles pas aussi des dimensions symboliques?

R.V.: Si bien sûr, mais pas les couches de coquilles qui recouvraient les corps... Dans la mesure du possible, on essayait de ne pas enterrer dans le sable. Dans les zones très sableuses où j'ai été amené à travailler, les habitants faisaient parfois de gros efforts pour aller chercher à distance des pierres à mettre sur les tombes. Mais un défunt pouvait être transporté assez loin, dans une zone pierreuse.

S.B.: Vous disiez que les corps étaient parfois enveloppés dans du cuir. Qu'est-ce qui vous permet d'avancer cela? R.V.: Il y a tous les modes possibles et imaginables d'inhumation: allongé ou sur le côté, dans des directions différentes, ou encore dans une position forcée, comme à Khatt Lemaiteg en position assise. Cela réclame alors un accessoire: un sac de tissu, de vannerie ou de cuir, une corde, ou, plus rarement, un coffrage en pierre. Mais dès que le défunt émerge du sable, le plus souvent à la suite d'un vent de sable, il peut se détruire en l'espace de quelques jours: il m'est arrivé à Khatt Lemaitteg de me retrouver au moment de repartir du site avec deux ou trois squelettes en position assise, parfois carrément avec le crâne posé sur les os des jambes. On sait déjà qu'il n'en restera au bout de quelques semaines que quelques os ou quelques esquilles. 
S.B.: Qu'est ce qui vous permet de dire que ces défunts étaient assis quand justement le mouvement des dunes peut changer la position d'un corps très facilement? R.V.: Le mouvement de la dune dans laquelle se situe l'inhumation est très limité. Ce sont les dunes superficielles qui peuvent écraser, et surtout se déplacer, laissant le corps exposé au vent, qui va excaver la tombe. Sur les photos, lorsque vous regardez la position des os, tibia, péroné et fémur sont " plantés » parallèlement, à peu près à la verticale : il n'y a guère de doute sur la position assise. Et la disposition du squelette en s'effondrant sur lui-même quand il n'est plus protégé par le sable et quand les liens, le tissu ou le cuir dans lequel il était enfermé, ont disparu, est caractéristique de la position assise, laquelle n'est pas rare dans le Néolithique mauritanien (El-Ghallaouia et Golfe d'Arguin) et plus généralement saharien.

M.-L.G.: Comment se fait-il que les grands ergs ne soient pas peuplés?
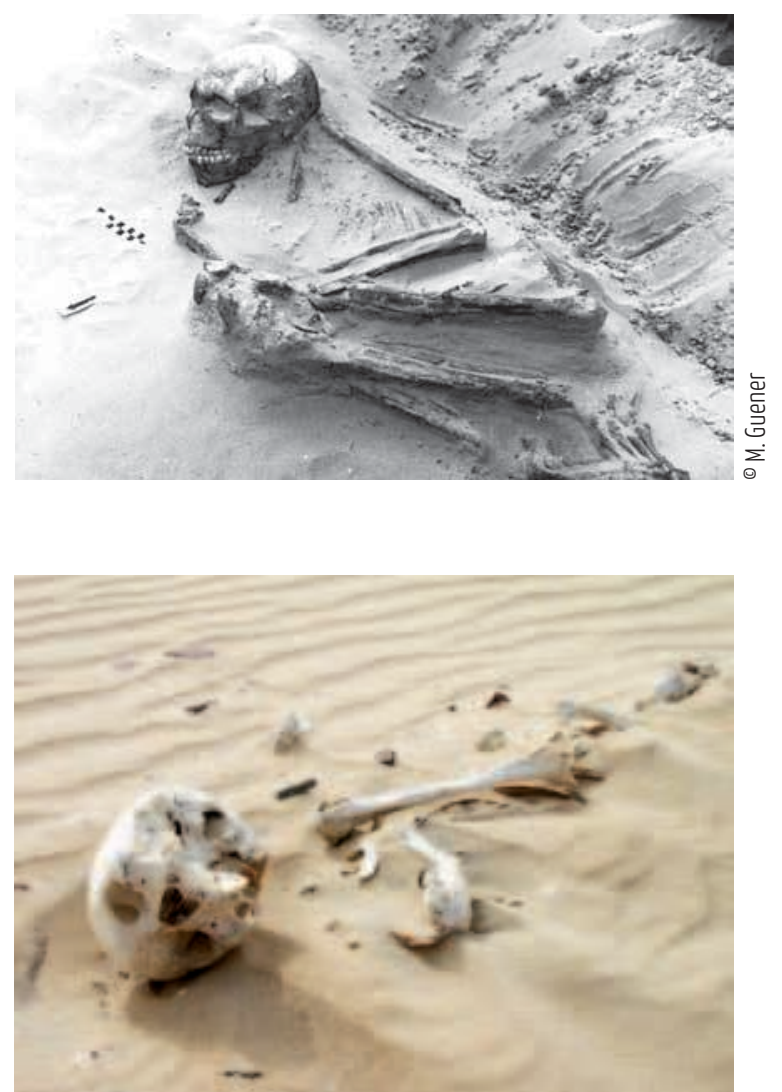

R.V.: Parce qu'il n'y a pas d'eau mais aussi parce que le sable est presque complètement azoïque. Dans une région où j'ai récemment travaillé, l'erg Ouarane, au nord-est de la Mauritanie, il existe aujourd'hui seulement quatre espèces végétales (Monod 1958). Au Groënland, il y en a cent cinquante. Certes, lorsqu'il pleut, il y a des millions de pousses, mais cela ne dure pas. C'est un milieu très particulier et impropre à la vie humaine en dehors des rares épisodes de pluie saisonnière.

M.-L.G. : Les archéologues s'intéressent-ils à ce qu'il y a sous le sable?

R.V.: Le sable saharien est relativement « casanier » : les grandes masses de sable bougent peu. Lorsqu'il y a une période sèche, même courte, le sable se remobilise mais il ne part pas, sauf granulométrie très fine (poussière) ou vent de sable terrifiant! Le grain de quartz d'un millimètre de diamètre reste sur place. Quand je suis arrivé à Nouakchott en 1974, il n'y avait pas de routes bitumées: on pouvait se rendre à Boutilimit (ville située à 150 km à l'est de la capitale)

\section{Une inhumation exceptionnellement bien conservée dans le sable, en decubitus latéral fléchi (Maïlima, nord de Nouakchott).}

Un cimetière d'époque historique sur la dune littorale au sud de Nouakchott (Chott Boul). Apparu peu de temps avant la photo, il avait disparu quelques semaines plus tard.

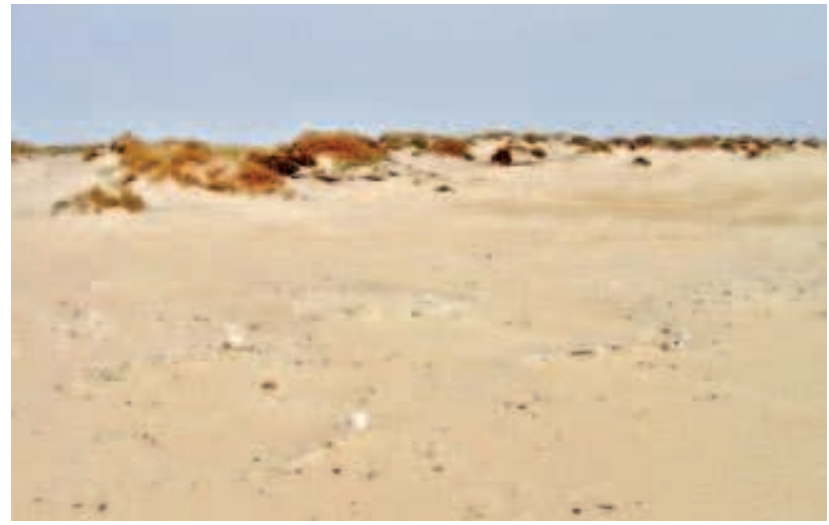


en Renault 4, simplement parce que la surface des cordons dunaires que nous devions traverser était dure. Que s'est-il passé ensuite? La construction et l'expansion de Nouakchott, à partir du milieu des années 1970, ont naturellement occasionné des déplacements de sable, ne serait-ce que par le piétinement des animaux, les voitures, et par l'exploitation de la végétation, alors encore assez dense, par l'homme. Mais ensuite, lors de la longue période de sécheresse des années 1970-1980, le vent a été suffisamment fort pour faire bourgeonner le sable en dunes vives de toutes sortes de formes, qui se sont progressivement fondues, à nouveau, dans les cordons plus anciens, lorsque la sécheresse s'est terminée. Cela signifie que, même il y a deux millions d'années, ces grands ergs étaient déjà en place et que l'on ne va rien trouver dessous. En revanche, s'il y a un, deux ou dix mètres de sable on peut espérer que la dune se déplacera un jour - ce qui est arrivé à peu près partout en Mauritanie occidentale, et a permis de découvrir des centaines de sites archéologiques.

M.-L.G.: On ne peut donc imaginer que du sable soit devenu de la terre ou qu'il y ait eu un déplacement? C'est une question importante car l'imaginaire de l'ensablement est très présent dans nos représentations du Sahara. Cela voudrait dire que les massifs dunaires se seraient peu déplacés?
R.V.: Le sable ne devient pas de la terre: il peut être recouvert d'un sol lors d'une période humide, mais, fondamentalement, il est là, quelle que soit sa forme. Pour que de grandes masses de sable se déplacent, il faut des conditions particulières de géologie, de topographie (un grand massif montagneux, comme le Tibesti ou l'Atlas saharien), qui fait dévier les vents et provoque de grands ergs, comme celui du Ténéré), des réseaux hydrographiques qui transportent et accumulent le sable, de grands espaces vides et plats où le sable peut fuser. La moindre différence topographique peut « accrocher » le sable, ou, au contraire, lui permettre de prendre son envol: les passages à travers les cordons dunaires correspondent en général à un léger bombement du relief, où le sable ne peut tenir, à cause de la fréquence et de la force du vent. Ce qui fait que l'occupation humaine se situe généralement sur les bords des ergs et dans les vallées interdunaires, surtout s'il y a de l'eau douce très près de la surface.

S.B.: Et donc, pour vous, qu'est-ce que cela implique dans votre pratique de l'archéologie?

R.V.: Mon expérience personnelle n'est sans doute pas la même que celle de chercheurs travaillant dans le Sahara central, dans les zones montagneuses en particulier, ou sur les regs du Sahara septentrional. Mais il est certain que dans

\section{Khatt Lemaïteg (Amatlich)} (3800-3000 calBP)

Un des cimetières du village, entre les dunes vives. On notera l'abondance des outils et débris de l'industrie en pierre et de la céramique. Divers modes d'inhumation : en médaillon : à gauche, decubitus latéral fléchi, avec une poterie funéraire à la tête ;

à droite : en position assise et forcée (dans un sac ?).
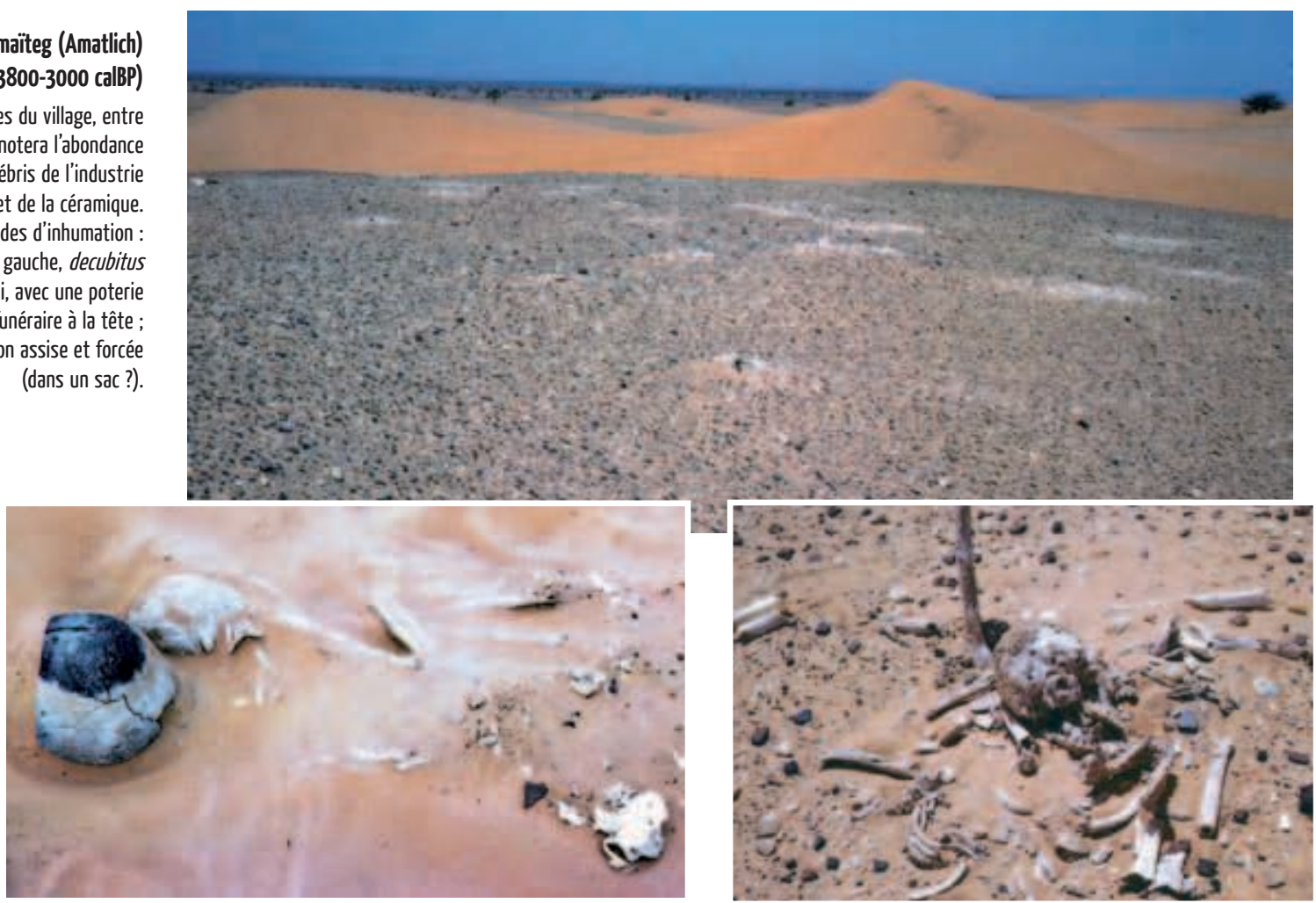


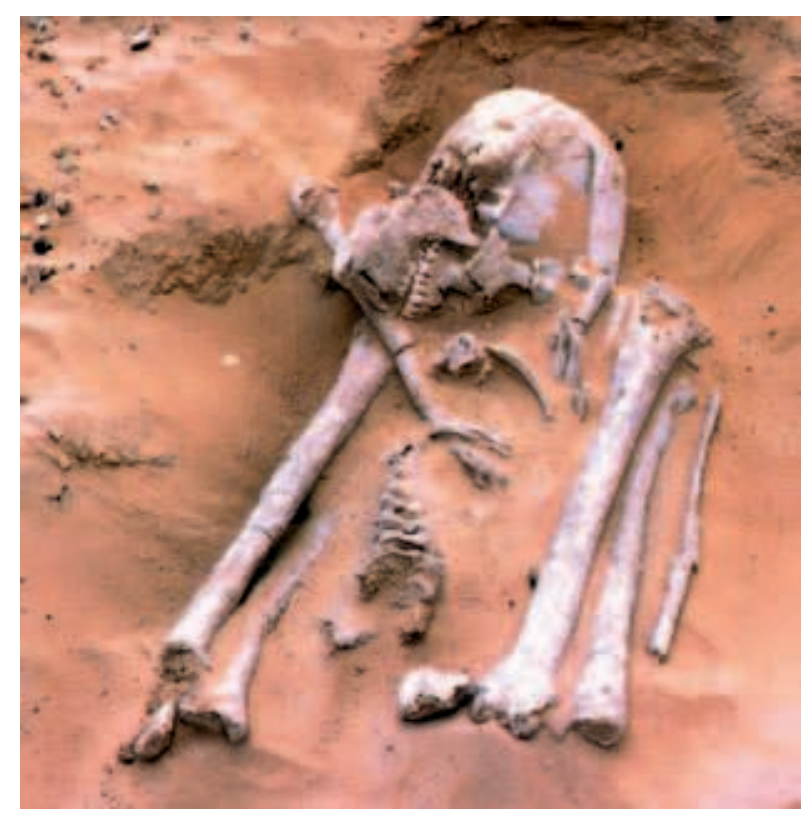

tous les sites importants en zone sableuse où j'ai travaillé, les sondages ont montré que le niveau archéologique ne dépassait guère $5 \mathrm{~cm}$ de profondeur, même si on va toujours à un mètre pour plus de certitude. Pour le Néolithique, aucun sondage ne m’a donné une stratigraphie, sinon dans des cas très particuliers d'amas coquilliers.

J'ai en revanche pu fouiller, sur le rivage atlantique nord, $25 \mathrm{~m}^{2}$ d'une butte sableuse occupée par deux sites, l'un épipaléolithique, l'autre néolithique et âgé de plus de 6000 ans. La couche supérieure est formée d'un voile coquillier de 10 à $20 \mathrm{~cm}$ d'épaisseur où l'industrie lithique, la céramique et les ossements animaux abondent. Sous la couche néolithique, $50 \mathrm{~cm}$ de sable stérile proviennent d'un épisode très aride bien connu, daté de plus de 8000 ans. Au-dessous, un certain nombre d'objets en pierre appartiennent à la culture épipaléolithique. Il s'agit d'une stratigraphie basique, le sondage ne nous ayant pas permis de trouver quoi que ce soit d'autre: en l'absence de matière organique dans la couche épipaléolithique nous n'avons pas de possibilité de datation. On notera que les habitats de cette culture se sont le plus souvent installés sur de fortes dunes isolées sur le reg, mais toujours du côté nordest, qui devait être protégé des vents les plus désagréables (venant donc du sud-ouest). En revanche, les néolithiques se sont installés de l'autre côté, à l'abri des vents de nordest. L'information paléoclimatique est très importante: le régime des vents a changé entre Holocène ancien et moyen, ce que confirment les travaux des spécialistes. Les hommes de l'Épipaléolithique ont vécu sur la dune ancienne, probablement vêtue, et y ont abandonné quelques outils, avant que leur habitat ne soit noyé dans une dune vive, puis que la période humide du Néolithique moyen n'apporte un sol sur lequel des collecteurs de mollusques marins se sont établis. Mais le voile de coquilles n'occupe pas toute la surface de la butte et, après l'installation définitive de l'aridité, le sol et la couche de sable ont été emportés par le vent. L'occupation épipaléolithique est aujourd'hui en surface partout où l'amas coquillier est absent. On a trouvé dans cette zone des milliers de pointes pédonculées - de sagaies plutôt que de flèches - et bien d'autres outils (Vernet et al. 2007). Mais un tel agencement est rare dans le sable et, par ailleurs, il est bien évident que la partie en stratigraphie du site épipaléolithique a été protégée par l'amas coquillier. On notera que la vision que nous avons aujourd'hui de la topographie ancienne et du mode d'installation des hommes à l'époque néolithique, est biaisée par l'évolution du sable au cours du temps.

S.B.: Un archéologue travaillant au Sahara n'est-il pas finalement toujours dans une archéologie d'urgence, d'opportunisme?

R.V.: Sauf financements importants (rares aujourd'hui!) ou présence de longue durée sur le terrain (un grand privilège!), on doit aborder ce terrain de manière très particulière. Il faut utiliser cet espace gigantesque (un million de $\mathrm{km}^{2}$ pour la Mauritanie) à la manière du carroyage des plus célèbres sites de la Préhistoire française, comme Pincevent, Tautavel ou l'abri Pataud! C'est-à-dire que là où l'on a un carroyage au mètre carré dans les zones non sableuses, la maille dans les zones sableuses sera, pour les grands espaces, d'un kilomètre. Certes on peut s'intéresser à des zones plus limitées, comme je l'avais fait à Nouakchott au début des années 1980 en travaillant sur des mailles de $250 \mathrm{~m}$ de côté. Mais cela peut aller jusqu'à cinq km. Pourquoi? Parce que le mode d'installation des hommes du Néolithique saharien est rarement sédentaire, sauf cas particuliers: les amas coquilliers en sont un, mais les hommes qui les constituaient étaient en général nomades; autre exemple, Khatt Lemaiteg, dont je parlais tout à l'heure, a été à un moment donné, au vu de sa taille, un site habité de façon permanente.

Hormis ces cas, on a des implantations qui sont soit saisonnières, soit aléatoires dans une zone de parcours donnée, pour des nomades chasseurs ou éleveurs. Ce sont donc, 

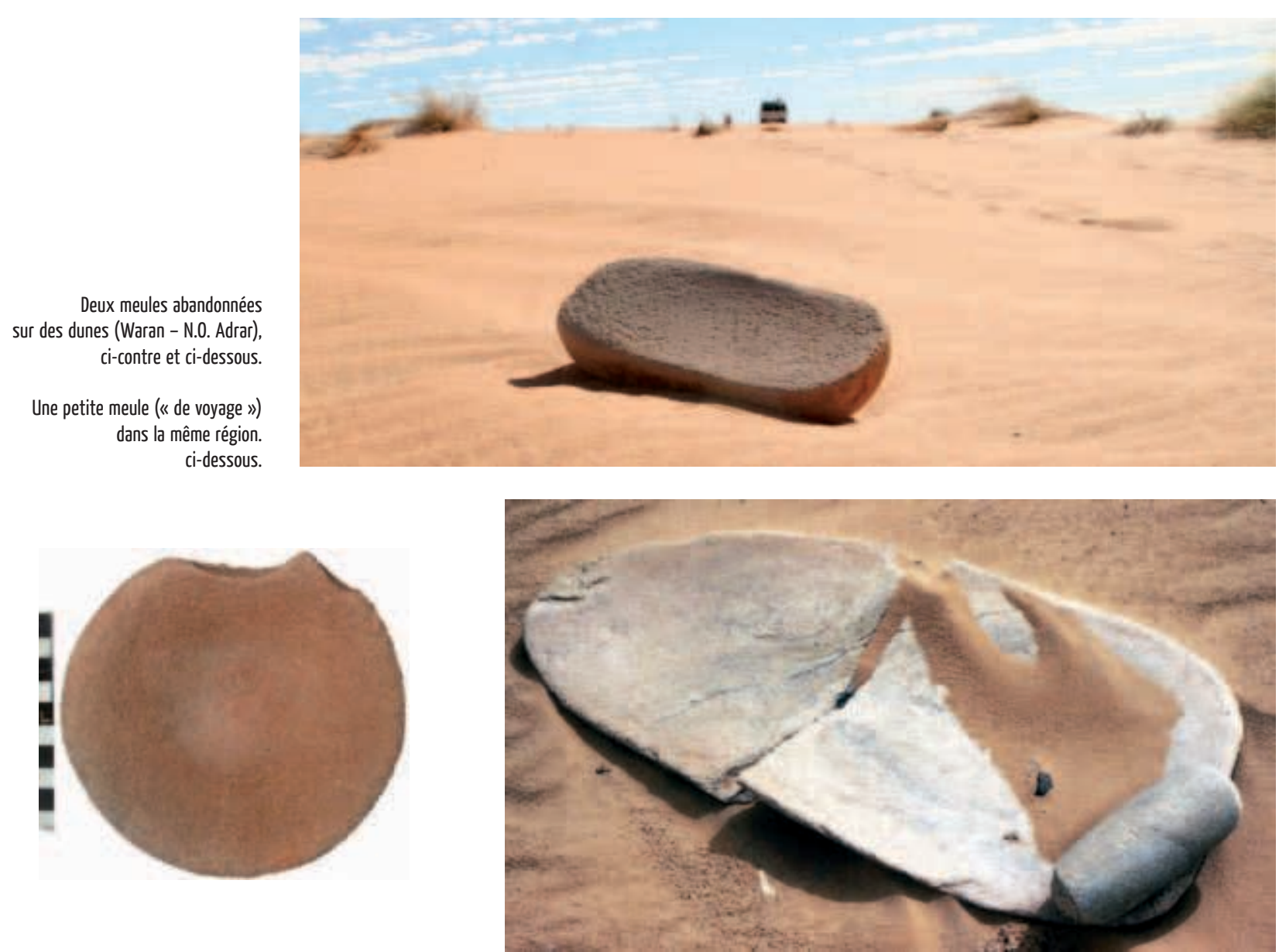

dans la plupart des cas, des sites très dispersés, souvent de petite taille, uniquement en surface, et ayant toujours été bousculés par le passage d'animaux, la pluie, le vent et la végétation. Peu d'objets sont restés intacts et en place. Depuis l'origine, beaucoup d'objets ont été pillés par des gens qui les récupéraient - et non, comme aujourd'hui, les vendaient ou les collectionnaient. Il faut donc trouver d'autres moyens que l'étude des sites et des objets en place pour se faire une idée du peuplement ancien de ces régions. Il est donc nécessaire de faire des prospections sur des zones très vastes. Plutôt que de partir d'un site ciblé que l'on va étudier à fond pour élargir ensuite au groupe humain concerné, on va partir de l'ensemble et essayer d'être de plus en plus précis, jusqu'à pouvoir fouiller un habitat essentiel de la culture. C'est la démarche que j'estime la plus efficace sur ce type de terrain, d'autant que les moyens humains et financiers qui sont les nôtres ne nous permettent guère autre chose. Bien entendu, lorsque les moyens sont réunis, comme à Ounjougou (Sahel malien), à Gobero (Ténéré), autour de certaines oasis du Western Desert égyptien ou dans le wadi Howar (Soudan), ce sont de véritables monographies qui peuvent être menées.
S.B. : Savez-vous si vos collègues archéologues du Sahara « sableux » suivent les mêmes principes de prospection que les vôtres et y a-t-il des échanges méthodologiques entre vous?

R.V.: Mais il n'y a malheureusement plus personne qui travaille sur ces terrains! Et de toutes les façons, il n'y a pas vraiment d'autres manières de travailler que celles que je vous ai décrites dans ces zones. Par exemple, le Ténéré du Niger n’a jamais vraiment été exploré archéologiquement parlant, faute de moyens de quadrillage.

Les projets de prospection pétrolière nous ont permis ces dernières années d'identifier 4000 nouveaux sites archéologiques en Mauritanie, sans compter les informations paléoclimatiques (lacs fossiles), dans une zone de $100000 \mathrm{~km}^{2}$ entre le Tagant et l'Adrar. La concession explorée par TOTAL était à $95 \%$ sableuse et très peu habitable parce que dépourvue de points d'eau accessibles. Il a fallu inventer la notion un peu étrange de «site néolithique minimum ». Dans ce type de région, on ne peut installer, même de manière saisonnière, un gros campement. Ce sont de toutes petites unités qui y sont allées, chasseurs ou bergers, seuls ou en famille. Que trouve-t-on sur 


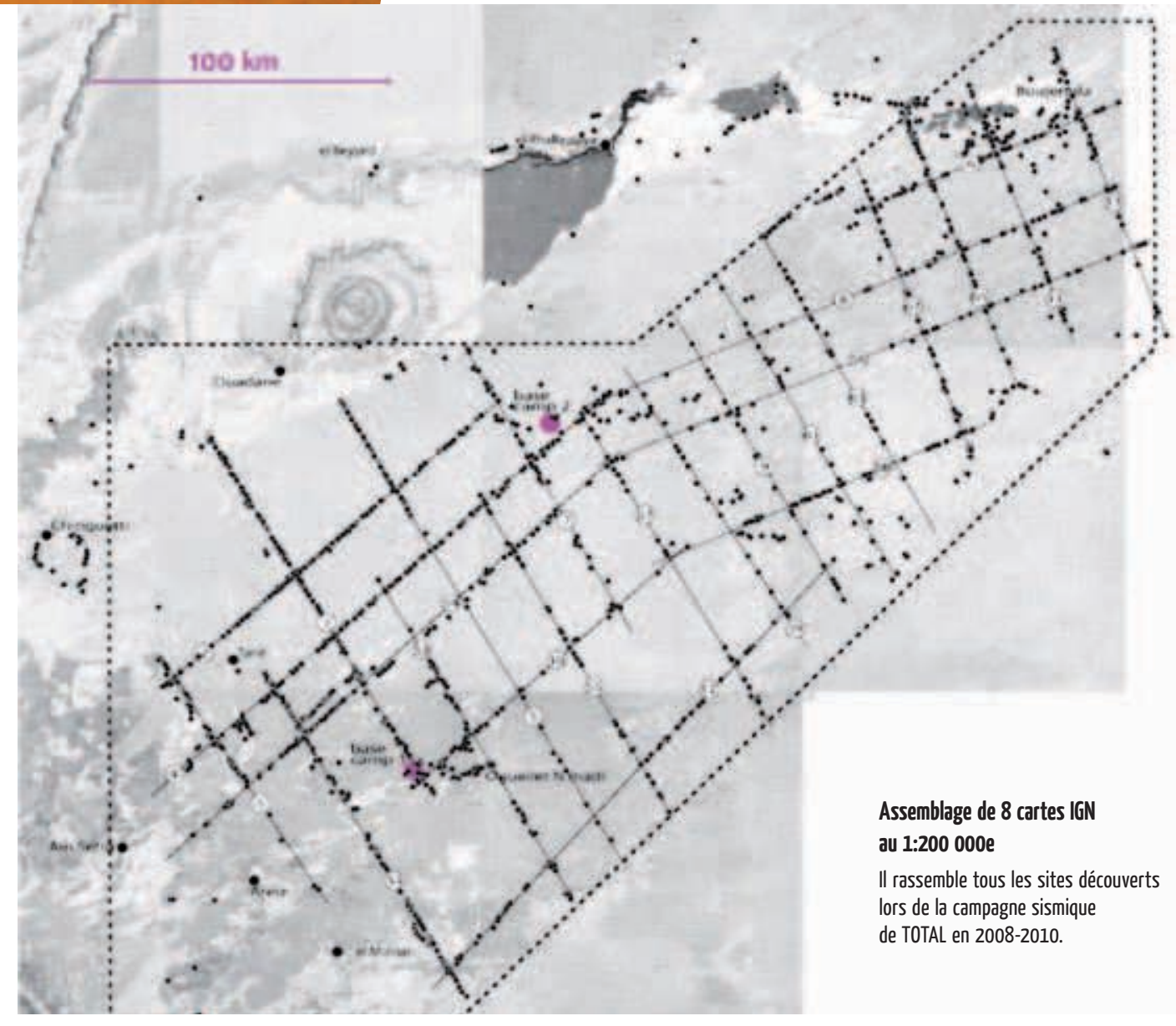

ces sites? Les nomades ont passé huit jours à un endroit, cassé une poterie ou une meule, mangé un addax ou une chèvre; ils sont ensuite partis vers un autre site. On a même identifié ce que j'appelle des «meules de voyage " ou «meules de poche », (fabriquées sur des fragments de meules classiques de cette période), plus petites et donc plus légères, et que l'on peut transporter plus facilement dans des zones où l'on se déplaçait beaucoup. (Figure 11) Donc ces notions curieuses de «sites minimums» ou d'« occupations minimales » sont vraiment liées aux vastes étendues sableuses.

S.B.: Dans le cadre de cette campagne d'archéologie préventive, comment avez-vous travaillé?

R.V. : Il est tout d'abord important de dire que l'on a travaillé pour un opérateur industriel. C'est-à-dire qu'on était soumis à ses attentes et qu'il attendait avant tout qu'on lui dise que tout allait bien et qu'il pouvait poursuivre son travail de prospection, sur des lignes droites de 20 à 100 kilomètres de long et de 200 mètres de large. Il va de soi que les archéologues essayaient de prospecter sur une largeur d'au moins un kilomètre. Mais au fond, le meilleur moyen de travailler dans ces espaces gigantesques (et compte tenu de nos moyens) était de travailler de manière aléatoire.

Nous avions peu de temps puisque l'archéologue était derrière le technicien en charge du traçage de la ligne de prospection sismique. Nous prenions simplement le référencement GPS des sites archéologiques et placions des petits drapeaux de couleur sur les sites repérés pour les protéger. Il est arrivé que nous fassions dévier le tracé de prospection pour protéger un site important.

S.B.: Une fois le site identifié, en quoi consistait votre travail?

R.V.: Cela se limitait a priori à cette mission d'inventaire des sites, de référencement et de prospection. Tout en sachant que les trois quarts des sites n'avaient qu'une importance scientifique limitée. Mais bien sûr, lorsque le site et les objets nous semblaient intéressants, voire exceptionnels, nous prenions notes et photographies, voire récoltions un ou quelques objets (tout a été déposé et enregistré à l'Institut Mauritanien de Recherches Scientifiques). Le tout encore une fois dans une urgence absolue. Le 
travail le plus important s'est fait après, à commencer par celui de la cartographie, puis l'étude des notes et du matériel. Quand vous avez noté cent cinquante fois que vous avez des haches fabriquées dans tel matériau, ou bien des pointes de flèches de telle forme, ou encore des meules qui ressemblent à celles de la «culture de Tichitt» plus méridionale, vous situez ces données sur votre carte et vous essayez d'en tirer de premiers enseignements. Dans la zone de prospection, nous avons fait vingt-neuf datations radiocarbone sur des tessons et avons réussi à identifier neuf ensembles céramiques clairement datés, nous permettant d'identifier la plupart les cultures ayant produit de la poterie dans la région.
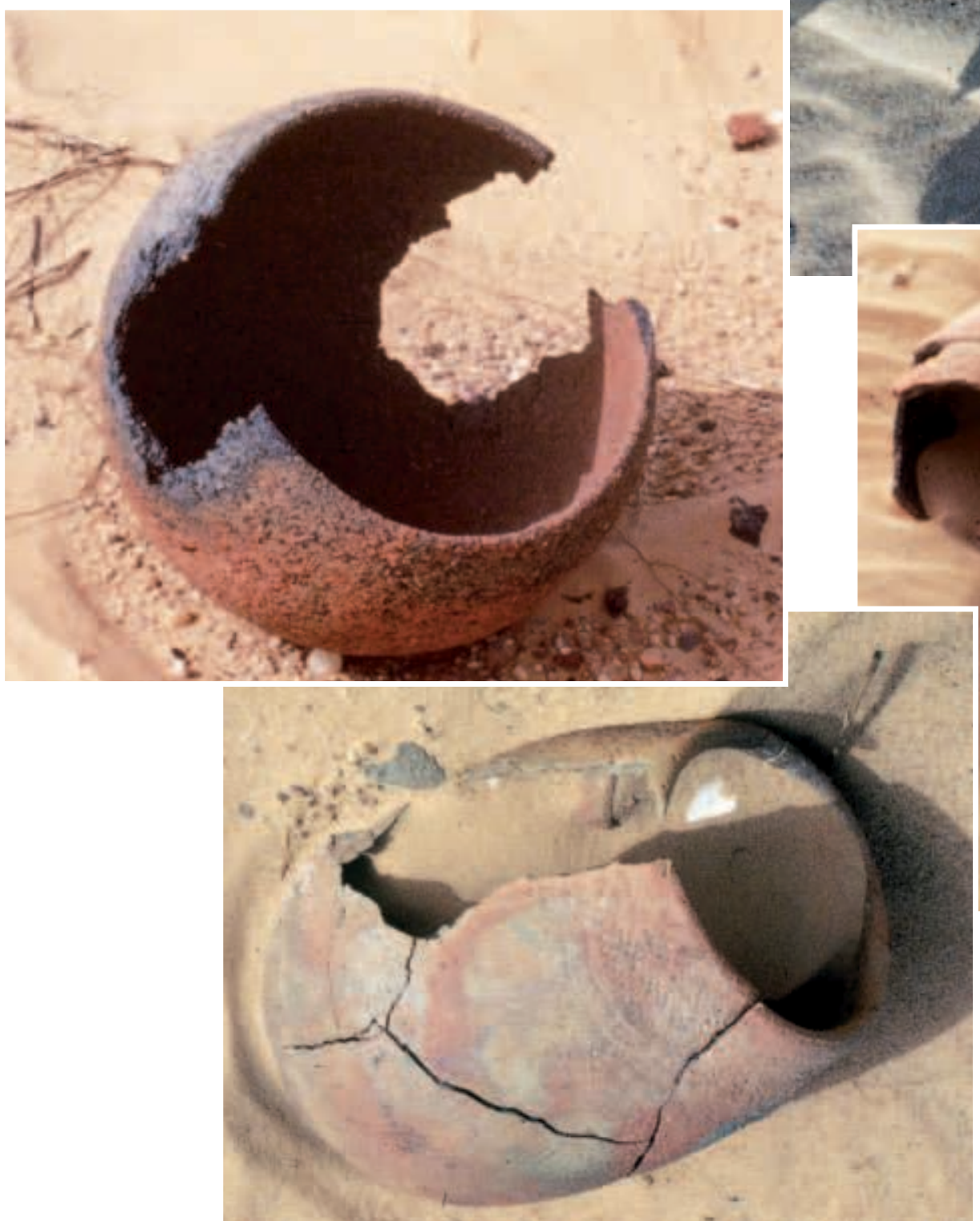

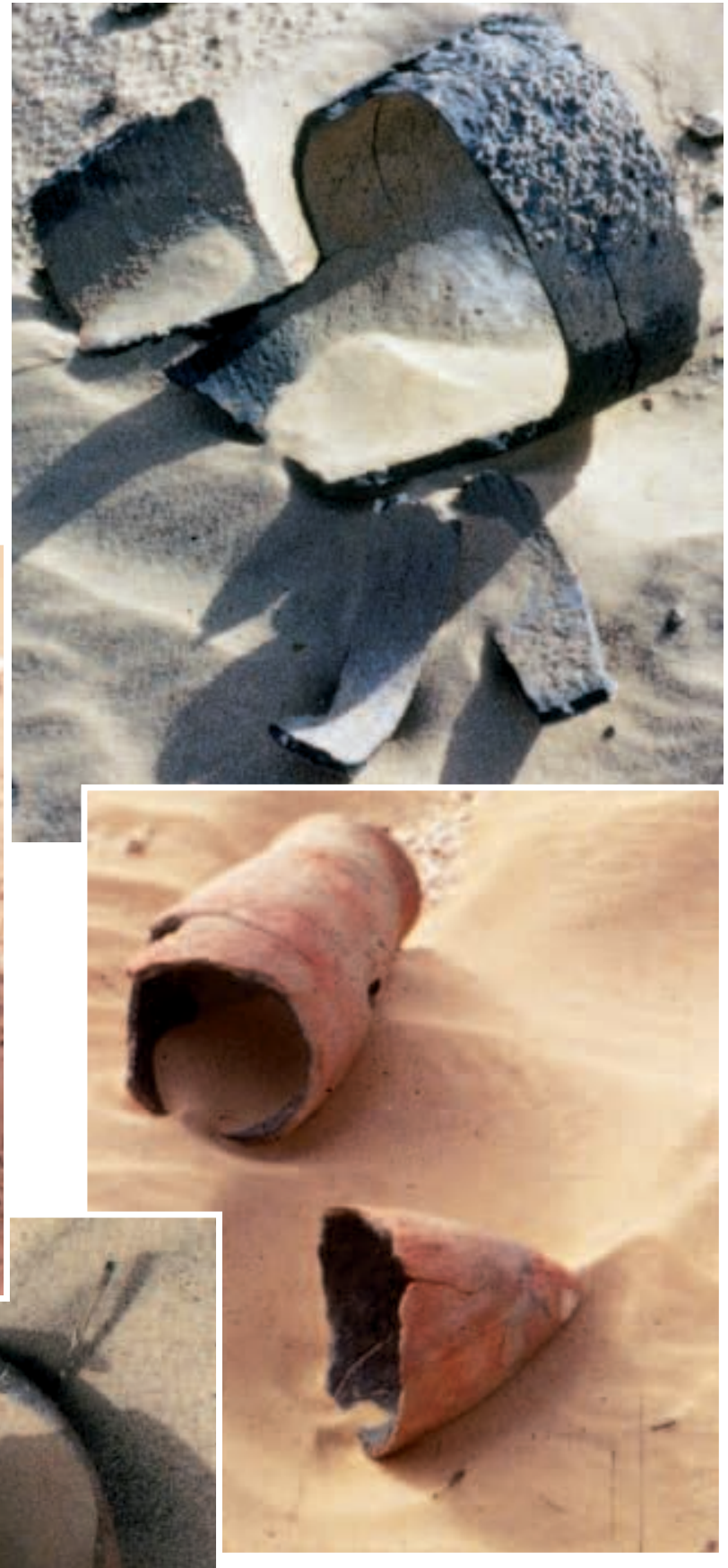




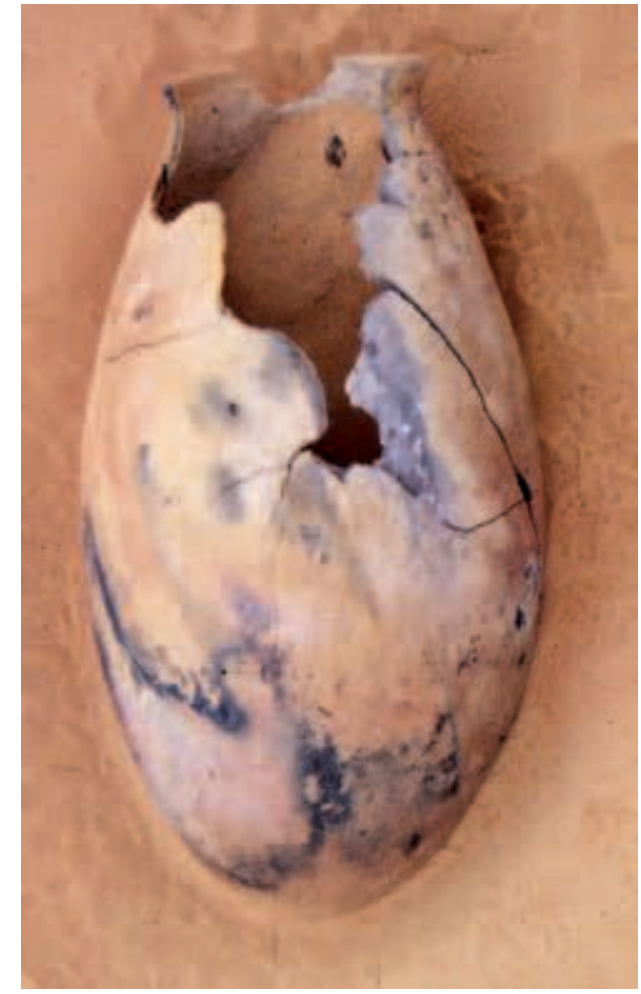

Le sable protège parfois les poteries, mais souvent les comprime et les ronge par l'alliance du vent et des grains de quartz.

Ici dans l'Ifozouiten, au N.E. de Nouakchott.
S.B.: Terminons par la question de la résistance, au temps et au sable, des différents matériaux utilisés par les hommes de la Préhistoire dans la fabrication de leurs objets...

R.V.: Si on évacue la notion de détérioration chimique (humidité, acidité, sel, dont l'action peut produire la disparition de la matière organique), le sable est surtout caractérisé par son rôle abrasif sur les autres matériaux, en particulier la céramique et la matière organique, surtout s'il est formé de grains de quartz très anguleux, dans une région, ou une période, très ventée. Il a ensuite un rôle d'enfouissement et, partant, il fait disparaître les objets (mais il ennoie aussi les paysages). Il écrase, mais il est aussi assez meuble. Je prends un exemple: une hache en pierre polie posée sur un sol sableux peut être engloutie sous une dune mobile - qui peut aussi se fixer ou disparaître. Mais si rien de ce type ne se produit, 5000 ans plus tard, la hache peut toujours être là - parce que le vent ne va pas bousculer la hache de pierre, mais va emporter les grains de sable qui l'entourent. Donc un outil en pierre ne « s'enterre » pas, mais reste toujours en surface, contrairement à ce qu'on peut imaginer. Et si le vent est toujours très fort, l'érosion va imprimer sa marque différemment, gommant, au vent, les détails de la fabrication de la pièce, qui seront conservés sous le vent.

Le sable est donc un élément fondamental dans le désert, qui dissimule et ronge, mais qui peut aussi faire réapparaître les paysages et les sites archéologiques. Au final, il protège les sites autant qu'il les menace...

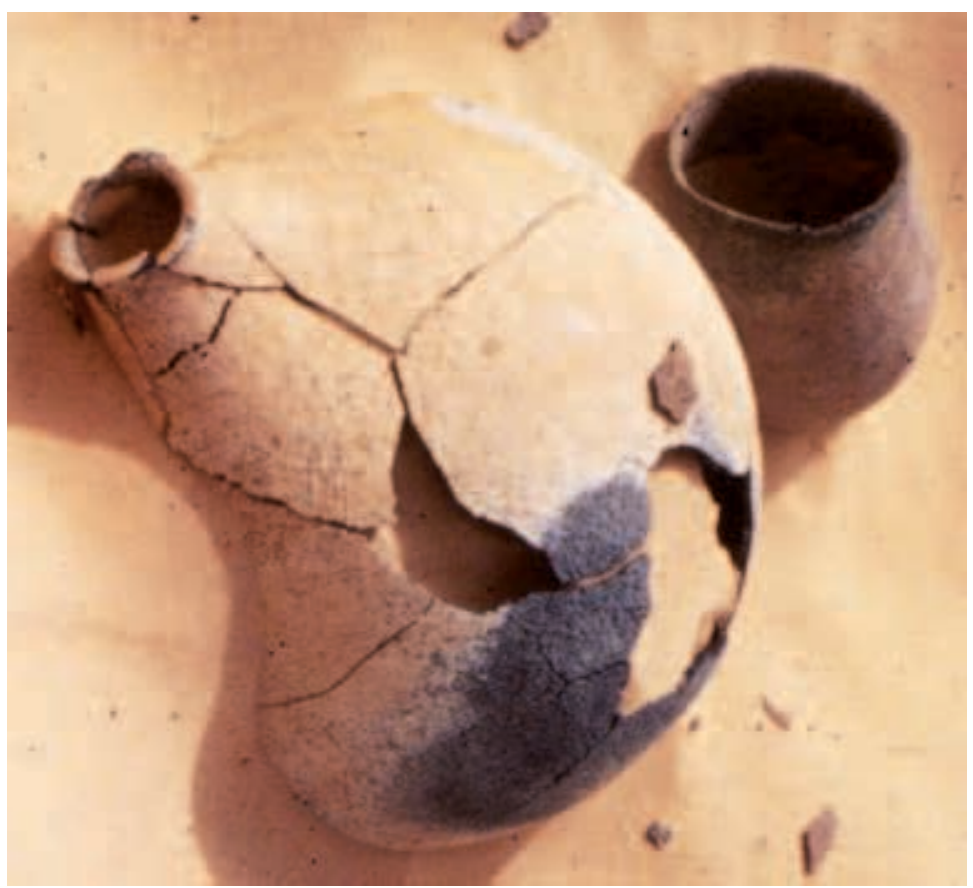




\section{NOTES}

Photo d'ouverture : Itilène, dans l'erg Waran (N.E. de l'Adrar).

Toutes les illustrations sont de l'auteur sauf mention contraire.

\section{RÉFÉRENCES}

Bathily, M.S., Ould Khattar, M. \& Vernet, R. 1998 Les Sites néolithiques de Khatt Lemaïteg (Amatlich) en Mauritanie occidentale. Nouakchott: CRIAA.

Di Lernia, S., Massamba N'siala, I. \& Mercuri, A.-M. 2012 Saharan prehistoric basketry. Archaeological and archaeobotanical analysis of the early middle Holocene assemblage from Takarkori (Acacus Mts., SW Libya), Journal of Archaeological Science 39/6: 1837-1853.

Heim, J.-L., Hadjaouis, D. \& Aumassip, G. 2011 L'Enfant néolithique (Homo 5) de Tin Hanakaten (Tassili des Ajjers, Algérie). Examen anthropologique et paléopathologique, Actes du Colloque international de Préhistoire maghrébine, Tamanrasset, Algérie, Travaux du CNRPAH 11 (N. S.), T. II : 27-40.

Khan Majliss, B. 1978 Der Nomadenhaushalt der Tuareg in Sahara : 10000 Jahre zwischen Weide und Wüste. [Handbuch zu einer Ausstellung des Rautenstrauch-Joest-Museums für Völkerkunde in Zusammenarbeit mit dem Institut für Ur- und Frühgeschichte der Universität zu Köln und dem Museum Alexander Koenig, Bonn], Museen der Stadt Köln : 365-369.

Maley, J. \& Vernet, R. 2013 Peuples et évolution climatique en Afrique nord-tropicale, de la fin du Néolithique à l'aube de l'époque moderne, Afriques [en ligne], 04 | 2013. URL: http://afriques.revues. org/1209 consulté le 03 février 2014.

Monod, T. 1958 Majâbat al Koubra, contribution à l'étude de l'« empty quarter » ouest saharien. Dakar: IFAN, mémoire $n^{\circ} 52$.

Sereno, P.C., Garcea, E.A.A., Jousse, H., Stojanowski, V.M., et al. 2008 Lakeside cemeteries in the Sahara: 5000 years of Holocene population and environmental change, PLoS ONE 3(8): 1-22.

Vernet, R. \& Boulanger, P. 2006 La céramique du cimetière de l'Aïn en Nouss en Mauritanie occidentale (3965 BP), in Préhistoire en Afrique, Actes du XIVe Congrès de l'UISPP, Université de Liège, Belgique, septembre 2001, BAR, Oxford: 119-126.

Vernet, R., Ott, M., Tarrou, L., Gallin, A. \& Géoris-Creuseveau, J. 2007 Fouille de la butte de FA 10 (Banc d'Arguin) et son apport à la connaissance de la culture épipaléolithique de Foum Arguin, nord-ouest du Sahara, Journal of African Archaeology 5(1): 17-45.

\section{POUR CITER CET ARTICLE}

Vernet, R., Boulay, S., Gélard, M.-L. 2014 Sable et préhistoire saharienne. Entretien avec Robert Vernet, in S. Boulay \& M.-L. Gélard, Vivre le sable! Corps, matière et sociétés, Techniques \& Culture 61 : 286-303. 


\section{RÉSUMÉ}

Le sable, l'homme de la Préhistoire et l'archéologue au Sahara. Le Sahara véhicule nombre d'idées reçues. Désert chaud, il n'est toutefois pas aride à toutes les époques. De même, il n'est pas une immensité sableuse : à 80 \% il est pierreux ou rocheux. Pourtant le sable y joue un rôle important, du moins dans certaines régions - en Mauritanie ou dans le Sahara méridional par exemple. Les populations néolithiques vivant dans les régions sableuses ont été confrontées à des contraintes matérielles particulières: l'eau, qui ne reste pas en surface; la matière première lithique qui est absente ou cachée; le sable et la poussière qui décolle au moindre vent, et dont il faut se protéger, les pratiques funéraires soumises à un substrat meuble et peu protecteur. L'évolution climatique joue un rôle clef: si l'apogée d'une période humide correspond à un pic de précipitations, pendant lequel le sable est dissimulé sous un sol riche et vêtu, le début et la fin de la période sont plus délicats. Les animaux domestiques le montrent bien, qui voient, lors du déclin d'un épisode humide, le petit bétail supplanter les bovins et, pour finir, le chameau devenir la pièce maîtresse de la survie dans le désert. Préhistoriens et paléoclimatologues subissent d'autres contraintes, liées à l'envahissement du paysage par le sable depuis quelques millénaires: accès difficiles aux zones de travail; enfouissement à long terme des restes archéologiques, mais aussi à court terme par les vents de sable; destruction des restes, en particulier organiques; immensité des espaces à inventorier... Tout cela implique des méthodes de travail adaptées, souvent dans l'urgence, et presque toujours opportunistes.

\section{ABSTRACT}

Sand, prehistoric man and the archaeologist in the Sahara. The Sahara conveys lots of generally accepted ideas. Hot desert, it has not always been arid in the past. While often considered as a sandy immensity, it is mostly (80\%) made of stones and rocks. Despite that, the sand occupies an important place, at least in certain regions - in Mauritania or in Southern Sahara for example. The Neolithic populations living in the sandy areas of the Sahara have been confronted with specific material constraints: water, which doesn't remain at the surface; the lithic material, which is absent or hidden; sand and dust often raised by the wind and from which one needs to protect itself; funerary practices submitted to a loose and under-protecting substratum. The climate evolution plays a key role. Whereas the apogee of a wet period corresponds to a peak of precipitations, during which the sand is covered by a rich and "clothed" ground, the beginning and the end of the period are more delicate. For example, during the decline of a wet period, small livestock supplants bovids and, at the end, camel becomes the main means to survive in the desert. Prehistorians and paleoclimatologists are subjects to other constraints linked with the invasion of landscapes by the sand since a few millenaries: difficult access to the research areas; long or short term burying of archeological vestiges by sand winds; relics'destruction, especially organic relics; immensity of the places and spaces to investigate... All these elements impose adapted research methods and imply to work in urgency and to conduct opportunist surveys.

\section{MOTS CLÉS}

Sable, Sahara, confort, Néolithique, archéologie, méthodes

\section{KEYWORDS}

Sand, Sahara, comfort, Neolithic, archeology, methods 